\title{
Investigation on Vibrations of a Cantilever Beam with Magnetorheological Fluid by Using the Acoustic Signal
}

\author{
J. Snamina $^{a, *}$, B. Sapiński ${ }^{a}$, W. WszoleK $^{b}$ And M. Romaszko ${ }^{a}$ \\ ${ }^{a}$ AGH University of Science and Technology, Faculty of Mechanical Engineering and Robotics \\ Department of Process Control, al. A. Mickiewicza 30, 30-059 Krakow, Poland \\ ${ }^{b}$ AGH University of Science and Technology, Faculty of Mechanical Engineering and Robotics \\ Department of Mechanics and Vibroacustic, al. A. Mickiewicza 30, 30-059 Krakow, Poland
}

\begin{abstract}
The paper presents results of laboratory investigations of a vibrating three-layer cantilever beam with magnetorheological fluid. The goal of the study was to determine changes of the acoustic field around the vibrating beam caused by modifications of the magnetorheological fluid properties. The experimental studies have been carried out on a dedicated measuring stand. The construction of the measuring stand allows fixing one end of the beam in a holder attached to the moving part of the electrodynamic shaker. The magnetic field is produced by an external electromagnet. During the measurements the beam displacements and the acoustic pressure have been simultaneously registered. Frequency analysis of the registered signals has been carried out in $1 / 12$ octave bands near the second natural frequency of the beam. The results reveal that the acoustic signal emitted by the vibrating beam decreases when the magnetic field is applied.
\end{abstract}

PACS: 46.40.-f, 46.70.De, 43.38.+n

\section{Introduction}

A sound emission is one of many processes accompanying vibrations of construction elements. Due to air properties on the surfaces of vibrating beams and plates acoustic waves are produced, which are able to propagate to the surrounding space and can be measured in an arbitrary point.

One of the methods for studying the vibration intensity of beams and plates is the measurement of acoustic field in their surrounding space. The sound intensity in any distance from the object is a result of the movements of all points of the vibrating beam and the acoustic properties of air. Thus the results of measurements are related to the movement of the whole object not only a selected point. In the case of continuous systems, such as beam, the final movement depends on natural modes contribution. Along the span of the considered object the vibration amplitudes change, depending on the boundary conditions and the location of excitation. The analysis of acoustic waves generated around beams executing vibrating motion has been extensively described in literature [1-3]. The papers are focused mainly on the noise reduction. Among other methods for reduction of vibrations and noise special piezoelectric elements are used, properly located on the surface of vibrating elements [4]. Recently, studies in many research centers are focused on three-layer beams and plates in which the internal layer is constituted by magnetorheological (MR) fluid $[5,6]$. Such fluids belong to a group of so-called intelligent mate-

* corresponding author; e-mail: snamina@agh.edu.pl rials, which modify their rheological properties under the action of an external magnetic field. The main parameters that can be modified are the stiffness and damping.

Because the changes take place in a very short time period (order of milliseconds) the MR fluids are excellent for application in systems characterized by high dynamics $[7,8]$. By generating an appropriate magnetic field one can minimize the vibrations of a plate or beam. An effective measure of the vibration intensity reduction is the measure of the sound intensity in the space around the beam or plate. The evaluation of beam vibrations using the acoustic measurements is based on the relation between averaged velocity of the points located on the surface of the beam and the acoustic pressure of the sound wave generated by the motion of the beam.

\section{Object of the study}

The examined object is a three-layer beam filled with MR fluid. The beam is formed by two aluminum strips $l=400 \mathrm{~mm}$ in length, $b=30 \mathrm{~mm}$ in width and $h_{1}=2 \mathrm{~mm}$ in thickness and the MR fluid (type 132DG manufactured by Lord Corporation [9]) layer, filling the space between the strips (see Fig. 1) [10]. The MR fluid is sealed by a spacer $g=1.5 \mathrm{~mm}$ in width and $h_{2}=2 \mathrm{~mm}$ in height made of silicon rubber. The influence of the MR fluid layer on stiffness and damping of the beam has been studied and described in papers [11, 12]. The beam vibrates in a magnetic field produced by an electromagnet (see Fig. 2). On both poles of the electromagnet coils have been wound, each consisting of 370 turns, made of copper wire $1.4 \mathrm{~mm}$ in diameter. There is a $\delta=20 \mathrm{~mm}$ distance between the poles of the electromagnet. 


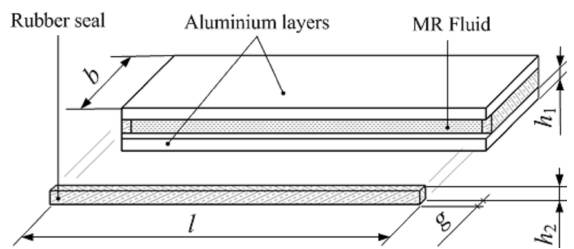

Fig. 1. Construction of the beam.

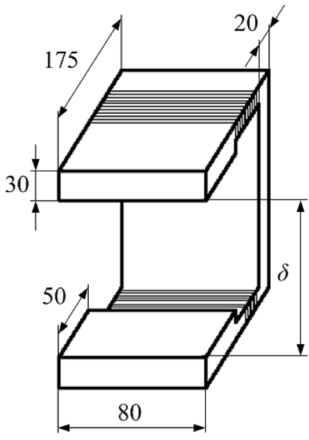

Fig. 2. Electromagnet layout.

\section{Measuring stand}

The measuring stand has been shown in Fig. 3. One of the beam ends has been clamped in a holder located at the moving part of the TIRA S514 electrodynamic shaker. The construction of the stand allows the movement of the electromagnet along the length of the beam. The electromagnet location is specified by the $y_{m}$ distance between the beam's fastening point and the mid-point of the electromagnet core. The electromagnet coil is supplied by DC current, produced by DF1731SB5A power supply.

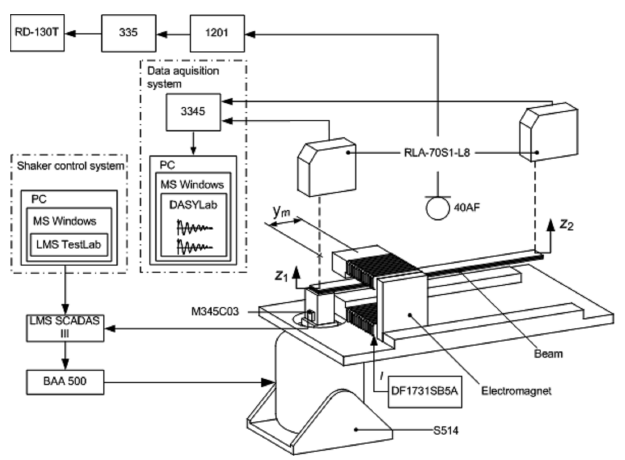

Fig. 3. Measuring stand.

The measuring stand is equipped with two measurement systems allowing the registration of the acoustic pressure level (sound intensity) and the beam vibrations in two points. The first point, in which the displacement has been registered, is the point where the beam is clamped to the shaker, and the second point is located at the free end of the beam. The $z_{1}(t)$ signal denotes the displacement of the moving part of the shaker (kinematic excitation) and the $z_{2}(t)$ signal denotes the displacement of the free end of the beam (the system's response). The displacement measuring system is made of two RLA-70S1-L8 laser beam converters, dedicated measurement card DAQPad 3345 by National Instruments and a PC computer with DASYLab software package. The registration of the acoustic pressure is executed by a system consisting of a $40 \mathrm{AF}$ measurement microphone made by G.R.A.S., 1201 microphone preamplifier and 335 signal amplifier, both made by Norsonic, and the RD-130T digital signal registrator (TEAC company). The moving part of shaker executes a motion in direction perpendicular to the beam axis. The shaker is controlled by the LMS SCADAS III module, programmed by a PC computer with a TestLab software. The control system of shaker motion uses also M345C03 piezoelectric accelerometer, made by PCB Piezoelectronics. The control signal is amplified by a TIRA BAA500 amplifier.

\section{Experiments}

The experiments have been carried out for a sinusoidally varying kinematic excitation, of constant frequency $f$ equal to $53.2 \mathrm{~Hz}[5]$. The frequency was chosen from the vicinity of the second natural frequency of the beam that was the lowest audible frequency in the set of natural frequencies. The amplitude of the shaker vibrations was $Z_{1}=0.7 \mathrm{~mm}$. The measurements have been performed for magnetic field strengths $H$ in the electromagnet air gap equal to 78 and $127 \mathrm{kA} / \mathrm{m}$. These values have been specified by the current $I$ flowing through electromagnet coil of 1 and $5 \mathrm{~A}$. In experiments the electromagnet position $y_{m}$ was equal to $100 \mathrm{~mm}$. The distance between the beam and measurement microphone was equal to $0.2 \mathrm{~m}$. In each experiment, the time histories have been registered for the beam vibrations: $z_{1}(t)$, $z_{2}(t)$ and the sound level. The $1 / 12$ octave frequency analyzer has been used for the noise and vibration analysis. The selected results are shown in Fig. 4 and Fig. 5. The plots in Fig. 4 present the measurement results for $I=1 \mathrm{~A}$, while the plots in Fig. 5 the measurement results for $I=5 \mathrm{~A}$.

During the measurements the shaker has been set on providing a sinusoidal displacement at frequency $f=53.2 \mathrm{~Hz}$ with the amplitude of $0.7 \mathrm{~mm}$. The excitation frequency is close to the second natural frequency of the beam. During the measurements the exciter generated vibrations were dominated by the harmonic motion with frequency of $53.2 \mathrm{~Hz}$. Additionally there were some extra harmonics generated with frequencies close to the dominating one, but with much smaller amplitudes. In the figures their levels (in relation to the main harmonic motion) are represented by negative values in the neighboring bands, close to the band in which the dominant frequency is located. The amplitude spectra of the excitation obtained by analysis using a set of $1 / 12$ octave 


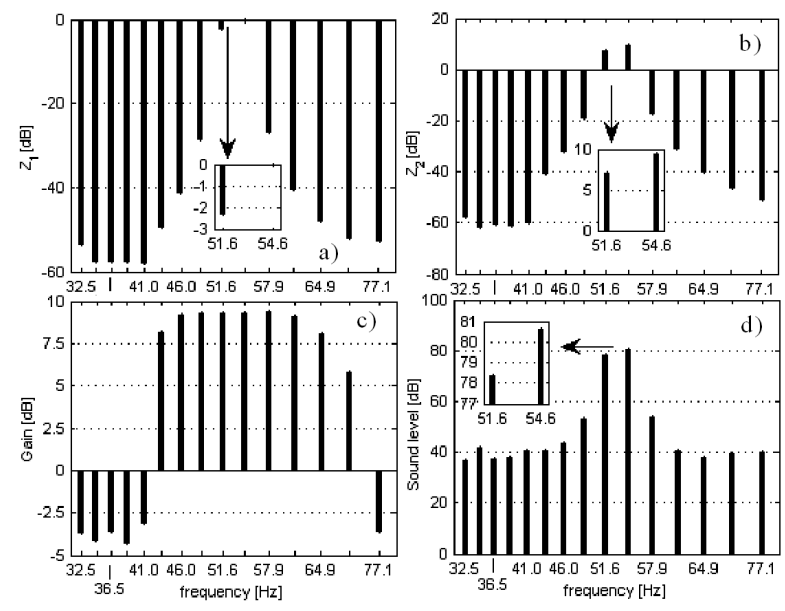

Fig. 4. Vibration and sound levels in selected bands of $1 / 12$ octave analyzer; $y_{m}=100 \mathrm{~mm}, f=53.2 \mathrm{~Hz}$, $I=1 \mathrm{~A}$.

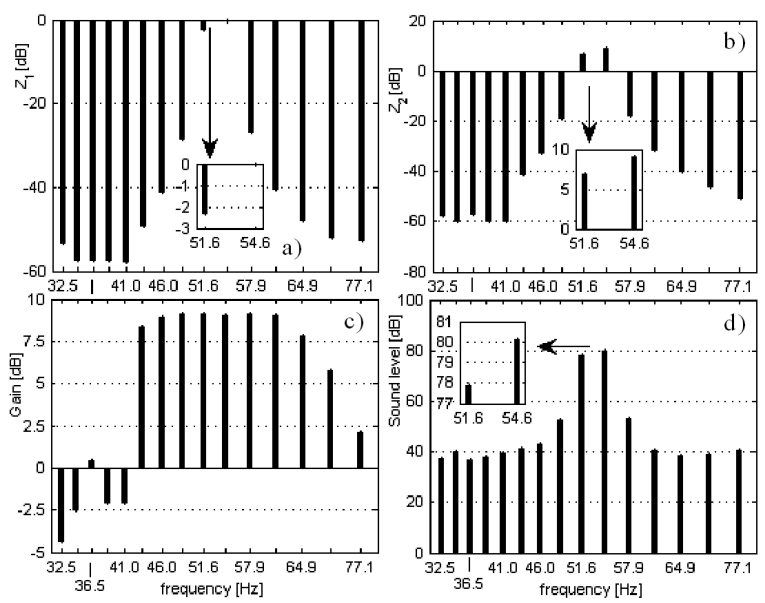

Fig. 5. As in Fig. 4, but for $I=5 \mathrm{~A}$.

filters are presented in Fig. 4a and Fig. 5a. During calculations of the vibration levels presented in these figures the reference amplitude has been taken as the amplitude set on the exciter, i.e. $0.7 \mathrm{~mm}$. The plots show that the vibration levels in the bands far from the set frequency of $53.2 \mathrm{~Hz}$ were considerably lower. Assuming the same conditions the frequency analysis has been carried out for the displacement at the free end of the beam. The results are presented in Fig. 4b and Fig. 5b. As expected the highest displacement amplitudes are found in the band covering the stimulation frequency and the band in its near vicinity. Using results obtained for the excitation signal $z_{1}(t)$ and the displacement signal at the beam's free end $z_{2}(t)$ the amplification has been calculated. The results are shown in Fig. 4c and Fig. 5c. They directly indicate a resonant nature of the beam vibration. The results of the sound level analysis for the sound emitted by the beam, calculated for the same bands of the 1/12 octave filter set, are shown in Fig. 4d and Fig. 5d. In the bands in which the highest sound level values are observed their values exceed by more than $10 \mathrm{~dB}$ the sound level of the acoustic background. During the measurements of the acoustic background sound level, the shaker, placed on the measuring stand, has been set on exactly the same parameters as during the measurements for the vibrating beam. The other elements located in the vicinity of the measuring stand have remained unchanged with respect to the situation in which the beam vibrations and the sound level have been measured.

\section{Conclusions}

The results of investigations show the influence of the MR fluid layer on the beam vibrations and the accompanying sound level. The influence of the MR fluid increases with increasing the current flowing through electromagnet winding. The MR fluid layer suppresses the vibrations leading also to the decrease of the sound level in the beam's neighborhood. The effect is small. For the highest applied value of the current $I=5$ A the resulting decrease of the sound level (in relation to case with the lowest value of the current $I=1 \mathrm{~A}$ ) was found to be $0.6 \mathrm{~dB}$. For lower current values the decrease of the sound level values was respectively lower. The obtained results are consistent with the corresponding results of the beam's vibration amplitudes.

\section{Acknowledgments}

The work has been completed as a part of the research project No. N501 223337.

\section{References}

[1] J. Pan, C.H. Hansen, J. Acoust. Soc. Am. 89, 200 (1991).

[2] W. Wszołek, R. Tadeusiewicz, Proc. Tenth Int. Congress on Sound and Vibration, Stockholm (Sweden), 2003, p. 709 .

[3] W. Wszołek, Mechanics 23, 317 (2004) (in Polish).

[4] R. Filipek, J. Wiciak, Europ. Phys. J. Special Topics 154, 57 (2008).

[5] Q. Sun, J.X. Zhou, L. Zhang, J. Sound Vibrat. 261, 465 (2003).

[6] V. Lara-Prieto, R. Parkin, M. Jackson, V. Siberschmidt, Z. Kęsy, Smart Mater. Struct. 19, 1 (2010).

[7] M. Yalcinitas, H. Dai, Smart Mater. Struct. 13, 1 (2004).

[8] Z.F. Yeh, Y.S. Shih, J. Comp. Mater. 40, 1333 (2006).

[9] http//www.lord.com, dated on: 24 IX 2011.

[10] B. Sapiński, J. Snamina, M. Romaszko, Techn. Trans. 2, 1-M/2011 (2011).

[11] B. Sapiński, J. Snamina, M. Romaszko, in: Proc. Vibrations in Physical Systems, Poznań-Będlewo (Poland), 2010, Vol. XXIV, Paper 50.

[12] J. Snamina, B. Sapiński, M. Romaszko, Eng. Model. 8, 39, 185, (2010). 